\title{
Detection of nanomechanical motion by evanescent light wave coupling
}

\author{
I. De Vlaminck ${ }^{\text {a) }}$ \\ IMEC, Kapeldreef 75, B-3001 Leuven, Belgium \\ J. Roels, D. Taillaert, D. Van Thourhout, and R. Baets \\ Ghent University-IMEC (INTEC), Sint-Pietersnieuwstraat 41, 9000 Gent, Belgium \\ L. Lagae and G. Borghs \\ IMEC, Kapeldreef 75, B-3001 Leuven, Belgium
}

(Received 13 December 2006; accepted 11 May 2007; published online 11 June 2007)

\begin{abstract}
The authors demonstrate a technique allowing sensitive nanomechanical motion detection based on the evanescent light wave coupling between two photonic nanowires. Any relative motion between the nanowires results in a change in light coupling, providing a means of registering motion. The in-plane vibrations of a $220 \mathrm{~nm} \times 400 \mathrm{~nm} \times 10 \mu \mathrm{m}$ nanomechanical resonator were recorded using this method. An analysis of the sensitivity reveals the potential of this integrated technique to provide fast and sensitive motion detection. (C) 2007 American Institute of Physics.
\end{abstract}

[DOI: $10.1063 / 1.2746067]$

Nanoelectromechanical systems (NEMSs) have received great attention in recent years. Most research has focused on resonant devices for sensor applications. ${ }^{1,2}$ Through scaling of the mechanical sensing element from the micron-to the nanoscale, researchers were able to improve the detection limits in force and mass sensing drastically. Devices sensitive enough to measure the magnetic moment of a single electron $\operatorname{spin}^{3}$ or to perform zeptogram scale mass sensing ${ }^{4}$ have resulted from efforts in scaling. A central problem in the development of even more sensitive mechanical sensors is the fast and high-precision detection of motion. In many cases, the performance of the sensor is restricted by the efficiency of the motion detection method employed, more so than by the inherent capabilities of the mechanical element. Much research has been devoted to solving this problem and a myriad of optical and electronic techniques has been developed. $^{5}$

Optical motion detection techniques have the inherent advantage that optical signals can be communicated with very large bandwidth. ${ }^{6-8}$ Unfortunately, for conventional optical techniques, diffraction effects limit the attainable detection limits when the mechanical devices are scaled. It has been anticipated that these issues can be overcome by integration of mechanical sensors with nanophotonics and by exploiting optical near-field effects. ${ }^{1,5}$ Recently, it was shown that motion of micron-scale devices can be monitored through changes in end-to-end alignment of optical waveguides. $^{9-11}$ In this letter, we propose an integrated and near-field optical motion detection technique that exploits near-field optical evanescent-wave coupling. We demonstrate that the technique works efficiently for nanoscale mechanical resonators, with greater sensitivity than conventional optical techniques.

The approach offers potential for applications where robust and sensitive measurement of motion is required and applications requiring remote detection or detection of motion in harsh environments. The integration of the device with photonic circuitry reduces the complexity of addressing large arrays of devices.

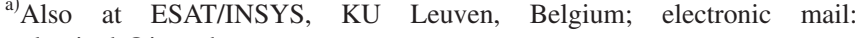
vlaminck@imec.be
The detection of nanomechanical motion is achieved through the near-field coupling of light from a photonic waveguide to a mechanical resonator. The mechanical resonator acts as a photonic waveguide; when the evanescent tails of the guided modes of the resonator and waveguide overlap, photons tunnel from one waveguide to the other. ${ }^{12}$ As described by the coupled-mode theory, the fraction of optical power exchanged is a function of the modal overlap. Hence, it depends on the coupling length, waveguide-towaveguide separation $s$, and amount of mode penetration. The sensitivity of power exchange to the separation between the waveguides provides a means of monitoring motion. If the interaction length is sufficient, total and coherent energy transfer can be achieved; therefore this principle can be exploited in the design of optical directional couplers. ${ }^{13} \mathrm{In}$ Fig. 1(a), the proposed sensing scheme is outlined. The light transmission past the freestanding structures, $P_{\text {out }} / P_{\text {in }}$, is the measured quantity. Both the main waveguide and the mechanical resonator are freestanding and doubly clamped. Electrodes are attached to the substrate waveguide and resonator; thus in- and out-of-plane motions can be actuated capacitively.

The structures were created in the silicon on insulator (SOI) material system. Silicon has attractive optical and mechanical properties. The high refractive index contrast between $\mathrm{Si}(n=3.48)$ and $\mathrm{SiO}_{2}(n=1.45)$ allows the design of submicron, low-loss, single-mode nanophotonic waveguides. ${ }^{14}$ The high stiffness and low mass density of $\mathrm{Si}(\rho$ $=2330 \mathrm{~kg} / \mathrm{m}^{3}$ ) are attractive for mechanical mass and force sensing. ${ }^{1}$ Furthermore, the technology can be integrated with Si-based electronics and mainstream fabrication techniques can be exploited.

The structures were fabricated in $p$-type, (100) SOI wafers (resistivity of $10 \Omega \mathrm{cm}$ ); the buried oxide has a thickness of $2 \mu \mathrm{m}$ and the top layer a thickness of $220 \mathrm{~nm}$. Deep ultraviolet lithography was used to define structures in the top silicon layer. ${ }^{14}$ Following the fabrication of the photonic structures, the wafers were carefully cleaned with a piranha solution $\left(\mathrm{H}_{2} \mathrm{SO}_{4} / \mathrm{H}_{2} \mathrm{O}_{2}\right.$ 4:1) and $\mathrm{Al} / \mathrm{Au}$ contacts were defined at the front and back sides of the wafer. Good contact resistances were achieved without annealing. In the last step 


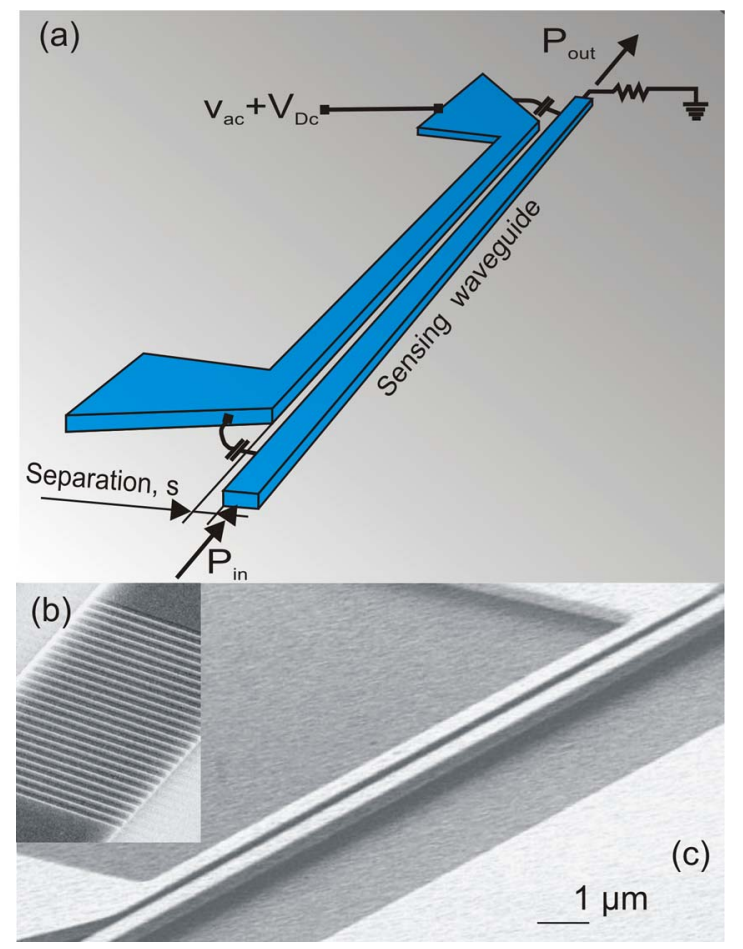

FIG. 1. (Color online) (a) Schematic drawing of the device under test. Any relative motion of the waveguide and resonator results in a change in output power $\left(P_{\text {out }}\right)$, providing a means of monitoring this motion. [(b) and (c)] Scanning electron micrographs of a one dimensional grating coupler (b) and of a typical device consisting of a mechanical resonator and a photonic waveguide (c).

a photo resist mask was defined and the structures were released by etching the oxide with buffered HF.

Figure 1(c) shows a scanning electron microscope (SEM) graph of a finished device (length of $10 \mu \mathrm{m}$ and width of $400 \mathrm{~nm}$ ). Optical attenuation values of $3.4 \mathrm{~dB} / \mathrm{mm}$ (Ref. 14) were recorded for waveguides fabricated with a similar process flow and similar dimensions. The waveguiderelated losses are dominated by scattering due to sidewall roughness.

Efficient coupling of light with a wavelength of $1550 \mathrm{~nm}$ from a single-mode fiber to the main waveguide and vice versa is realized by means of a one dimensional (1D) grating coupler and a tapered waveguide. ${ }^{15}$ Figure $1(\mathrm{~b})$ shows a SEM picture of a one dimensional grating structure used for light coupling to the chip. Coupling efficiencies up to $30 \%$ per coupler can be achieved for this type of coupling structure. $^{15}$

The optical measurements on the fabricated devices are taken at room temperature and at ambient conditions. In Fig. 2(a) the experimental setup is outlined. Light $(1550 \mathrm{~nm})$ from a tunable laser is guided to the sample with optical fibers and coupled in a nanoscale optical waveguide via $1 \mathrm{D}$ coupling structures and parabolic tapered waveguides. The light transmission is measured with a fast detector $(D)$; electrical signals are amplified with a low noise amplifier (LNA) and are spectrally analyzed with a network analyzer (NA). In Fig. 2(b), the resonant response of the fundamental in-plane mode of the mechanical resonator is shown. The resonance frequency, $f_{\text {res }}=\omega_{\text {res }} / 2 \pi$, for the beam with dimensions $t$ $\times w \times l=220 \mathrm{~nm} \times 400 \mathrm{~nm} \times 10 \mu \mathrm{m}$ (with $w, t$, and $l$ the width, thickness, and length of the resonator) is $27.6 \mathrm{MHz}$, $s=300 \mathrm{~nm}$. A quality factor $Q$ of 150 was extracted by LorDownloaded 26 Jul 2007 to 157.193.172.109. Redistribution subject

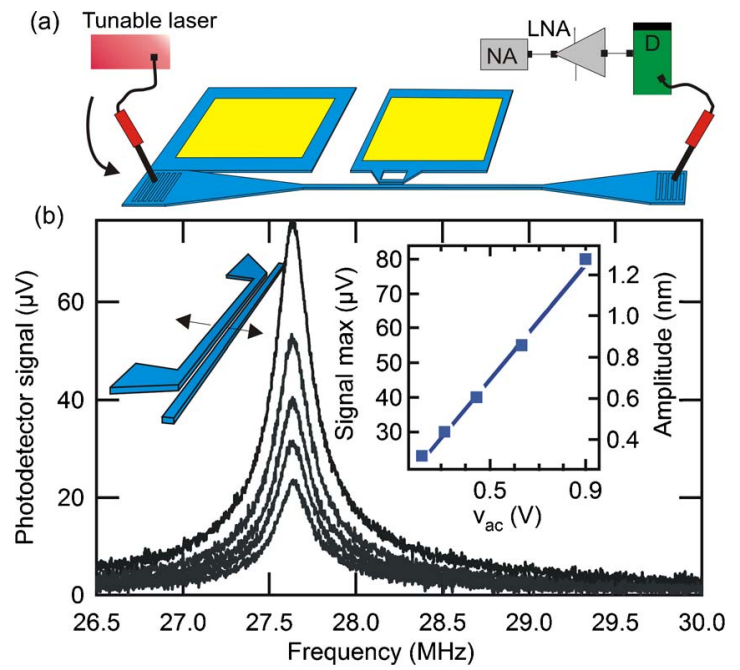

FIG. 2. (Color online) (a) Sketch of the setup. Laser light, $\lambda=1550 \mathrm{~nm}$, is guided by optical fibers and coupled into the 1D grating on the sample. Transmitted light is collected and fiber guided to a fast optical detector $(D)$. The electrical signal is amplified (LNA) and read out by the signalgenerating NA. (b) Measured resonance vibration spectrum for a beam with dimensions $t \times w \times l=220 \mathrm{~nm} \times 400 \mathrm{~nm} \times 10 \mu \mathrm{m}$; the ac voltage was stepwised increased (see inset).

entz fitting the vibration spectrum. Air damping of the mechanical motion is the dominant energy loss mechanism. The laser power $P_{\text {laser }}$ applied in the experiment was $5 \mathrm{~mW}$; no measurable influence of the optical power on the spectral shape nor on the resonance frequency was found for optical powers up to $7.5 \mathrm{~mW}$, the maximum power of the laser. $50 \mu \mathrm{W}$ of optical power was collected at the input of the optical detector. A $2 \mathrm{~V}$ electrical dc-bias voltage $V_{b}$ was applied. The applied ac voltage, $v_{\mathrm{ac}}$ was stepwise increased, leading to a linear increase in vibration amplitude and accordingly power modulation. As an indication, we plot the photodetector signal and vibration amplitude at resonance corresponding to the different drive voltages. The vibration amplitude was calculated as $x\left(\omega_{\text {res }}\right)=Q C /(k s) V_{b} v_{\text {ac }}\left(\omega_{\text {res }}\right)$, with $k$ the beam stiffness, $k=M_{\text {eff }} \omega_{\text {res }}^{2}$ (with $M_{\text {eff }}$ the effective mass, $\left.M_{\text {eff }}=0.73 \rho w t l\right)$ and $C$ the drive capacitance, $C=\varepsilon_{0} l t / s$ (with $\varepsilon_{0}$ the vacuum permittivity). Note that different mechanical vibration modes, in plane and out of plane, fundamental and higher harmonic, of both the mechanical resonator and the freestanding part of the photonic waveguide can be excited and monitored. In this letter, we focus on the important, fundamental in-plane mode of the mechanical resonator.

To assess the sensitivity of light coupling to waveguideresonator displacements, a three dimensional full-vectorial three dimensional mode solver was employed. In Fig. 3(a) the mode solutions for $s=150$ and $300 \mathrm{~nm}$ are plotted and compared. Clearly, the light coupling is significantly stronger for the smaller separation case. We define the responsivity of the system $\mathfrak{R}_{0}$ as follows:

$$
\mathfrak{R}_{0}=\left.\frac{1}{P_{\text {in }}} \frac{\partial P_{\text {out }}}{\partial s}\right|_{s=s_{0}} .
$$

In Fig. 3(b) the transmission, $T=P_{\text {out }} / P_{\text {in }}$, and $\Re_{0}$ are plotted as function of the in-plane waveguide separation. The dimensions of the resonator were chosen to be $220 \mathrm{~nm}$ $\times 400 \mathrm{~nm} \times 10 \mu \mathrm{m}$. The width and thickness of the main waveguide are the same, maximizing the coupling efficiency. to AIP license or copyright, see http://apl.aip.org/apl/copyright.jsp 

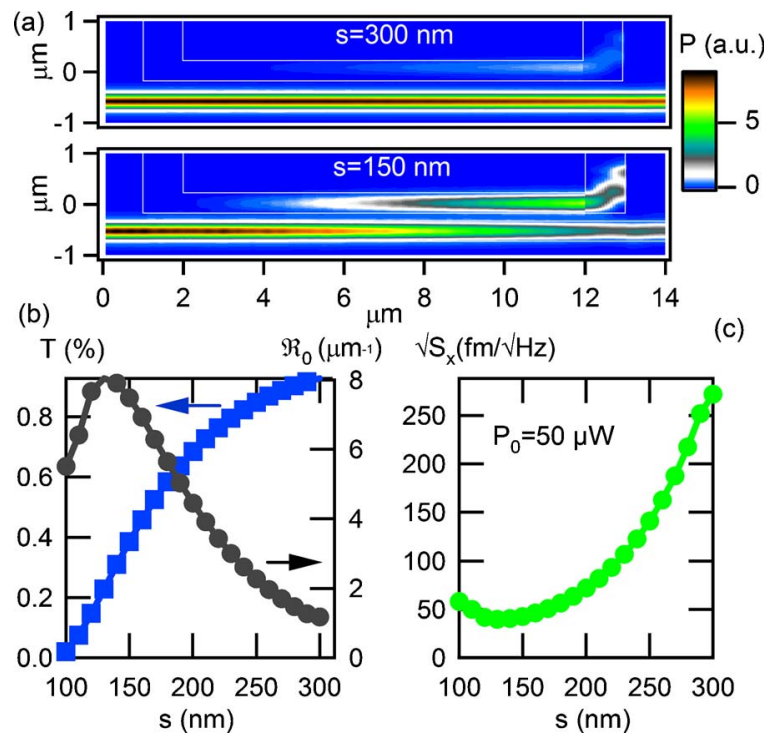

FIG. 3. (Color online) (a) Comparison of the mode solution for gaps of 300 and $150 \mathrm{~nm}$; more power $(P)$ is coupled to the mechanical resonator for the smaller gap case $(t \times w \times l=220 \mathrm{~nm} \times 400 \mathrm{~nm} \times 10 \mu \mathrm{m})$. (b) The transmission of power, $T=P_{\text {out }} / P_{\text {in }}$, as function of in-plane separation and the sensitivity to displacements $R_{0}$ are shown. (c) The displacement resolution, ${ } S_{x}$, as function of $s$.

The $\mathfrak{R}_{0}$ values are in the range of $10 \mu \mathrm{m}^{-1}$, higher than the responsivity achieved with interferometric techniques probing similar sized resonators. In comparison, $\mathfrak{R}_{0}$ values in the range of $0.2 \mu \mathrm{m}^{-1}$ were reported recently for an optical technique applicable for in-plane vibration detection, ${ }^{6}$ and $0.01 \mu \mathrm{m}^{-1}$ for an optical technique applicable for out-ofplane vibration detection. ${ }^{7}$ Note that influences of beam bending were disregarded in the analysis of the responsivity.

In general, an enhanced responsivity can be expected for improved modal overlap for the two waveguides. Figure 3(b) illustrates the importance of small spacing between the waveguides in this context, also indicating the importance of a high resolution fabrication process. Decreasing the width of the waveguide structures helps increase the portion of optical power in the evanescent part of the optical mode and hence increase the responsivity (in order to reduce the related increase in power loss, one might want to reduce the width of the sensing waveguide only in the coupler region).

A more drastic improvement may be expected for more fundamental changes in design. For example, coupling nanomechanical resonators to on-chip high- $Q$ cavities such as ring resonators could be employed for displacement sensing with great sensitivity. ${ }^{18}$

We now estimate the attainable displacement resolution, $\sqrt{ } S_{x} \cdot \sqrt{ } S_{x}$ can be expressed as

$$
\sqrt{S_{x}}=\frac{\mathrm{NEP}}{\alpha P_{\text {laser }} \Re_{0}},
$$

with $\alpha$ the optical transmission through the fibers and coupling structures in the setup and NEP the noise equivalent power related to the noise sources in the measurement scheme referred to the input of the photodetector.

In Fig. 3(c), we plot $\sqrt{ } S_{x} . P_{\text {laser }}$ was taken to be $5 \mathrm{~mW}$ and $\alpha$ is 0.01 . We take into account the NEP of the optical detector, $15 \mathrm{pW} / \sqrt{ } \mathrm{Hz}$. In this case, our detection method with high $\mathfrak{R}_{0}$ leads to a $\sqrt{ } S_{x}$ in the $50-350 \mathrm{fm} / \sqrt{ } \mathrm{Hz}$ range for $s$ in the range of 100-300 nm.
A higher responsivity only leads to a better performance in sensing applications when the linear dynamic range is not adversely affected ${ }^{1}$ by additional sources of noise or instabilities or by nonlinear phenomena. Nonlinearities can limit the maximal optical power modulation through bifurcation, distortion, and noise aliasing effects. ${ }^{1,16}$ The strong dependence of transmission on waveguide separation quoted makes our system more prone to noise aliasing and distortion induced by the optical transduction. The transduction technique only affects the maximum optical power modulation when optical-induced nonlinearities dominate the mechanical and electrical nonlinear effects. The optical power modulation is proportional to the applicable optical power. Note in this context that heating of the structures through light absorption is very small at $\lambda=1550 \mathrm{~nm}$. Optomechanical coupling is possible through optical forces due to interacting induced dipole moments ${ }^{17}$ and through radiation pressure exerted by photons on the beams. ${ }^{18}$

The strong coupling between identically dimensioned, parallel waveguides leads to a high displacement sensitivity. For those situations where the requirement of parallel waveguides is too stringent, evanescent coupling to a nearby surface or scatterer could be considered for motion detection.

In conclusion, we have demonstrated that nanomechanical motion can be detected with high sensitivity and high bandwidth by exploiting the strong waveguide separation dependence of evanescent optical coupling between two nanophotonic waveguides. The results clearly show the potential of optically integrated NEMS.

The authors thank Johan Feyaerts and Erwin Vandenplas for technical support. Two of the authors (I.D.V. and D.T.) acknowledge financial support from the I.W.T. (Flanders).

${ }^{1}$ K. L. Ekinci and M. L. Roukes, Rev. Sci. Instrum. 76, 061101 (2005).

${ }^{2}$ I. De Vlaminck, K. De Greve, L. Lagae, and G. Borghs, Appl. Phys. Lett. 88, 063112 (2006).

${ }^{3}$ D. Rugar, R. Budakian, H. J. Mamin, and B. W. Chui, Nature (London) 430, 329 (2004).

${ }^{4}$ Y. T. Yang, C. Callegari, X. L. Feng, K. L. Ekinci, and M. L. Roukes, Nano Lett. 6, 583 (2006).

${ }^{5}$ K. L. Ekinci, Small 1, 786 (2005).

${ }^{6}$ D. Karabacak, T. Kouh, C. C. Huang, and K. L. Ekinci, Appl. Phys. Lett. 88, 193122 (2006).

${ }^{7}$ T. Kouh, D. Karabacak, D. H. Kim, and K. L. Ekinci, Appl. Phys. Lett. 86, 013106 (2005).

${ }^{8}$ B. E. N. Keeler, D. W. Carr, J. P. Sullivan, T. A. Friedmann, and J. R. Wendt, Opt. Lett. 29, 1182 (2004).

${ }^{9}$ E. Ollier, IEEE J. Sel. Top. Quantum Electron. 8, 155 (2002).

${ }^{10}$ M. W. Pruessner, N. Siwak, K. Amarnath, S. Kanakaraju, W.-H. Chuang, and R. Ghodssi, J. Micromech. Microeng. 16, 832 (2006).

${ }^{11}$ K. Zinoviev, C. Dominguez, J. A. Plaza, V. J. C. Busto, and L. M. Lechuga, J. Lightwave Technol. 24, 2132 (2006).

${ }^{12}$ R. G. Hunsperger, Integrated Optics: Theory and Technology, 3rd ed. (Springer, Berlin, 1991), Vol. 33, pp. 110-123.

${ }^{13}$ M. W. Pruesnner, K. Amarnath, M. Datta, D. P. Kelly, S. Kanakaraju, P.-T. Ho, and R. Ghodssi, J. Microelectromech. Syst. 14, 1070 (2005).

${ }^{14}$ W. Bogaerts, R. Baets, P. Dumon, V. Wiaux, S. Beckx, D. Taillaert, B. Luyssaert, J. Van Campenhout, P. Bienstman, and D. Van Thourhout, J. Lightwave Technol. 23, 401 (2005).

${ }^{15}$ D. Taillaert, F. Van Laere, M. Ayre, W. Bogaerts, D. Van Thourhout, P. Bienstman, and R. Baets, Jpn. J. Appl. Phys., Part 1 45, 6071 (2006).

${ }^{16}$ V. Kaajakari, J. K. Koskinen, and T. Mattila, IEEE Trans. Ultrason. Ferroelectr. Freq. Control 52, 2322 (2005).

${ }^{17}$ M. L. Povinelli, M. Loncar, M. Ibanescu, E. J. Smythe, S. G. Johnson, F. Capasso, and J. D. Joannopoulos, Opt. Lett. 30, 3042 (2005).

${ }^{18}$ M. Hossein-Zadeh, H. Rokhsari, A. Hajimiri, and K. J. Vahala, Phys. Rev. A 74, 023813 (2006). 